Article

\title{
Antagonist Muscle Co-Activation during Kettlebell Single Arm Swing Exercise
}

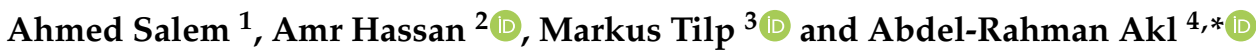 \\ 1 The International Academy of Sports Science and Technology (IASST), The Greek Campus, \\ Cairo 11513, Egypt; ahmedsalem731@gmail.com \\ 2 Department of Sports Training, Faculty of Sports Education, Mansoura University, Mansoura 35516, Egypt; \\ amrahh@mans.edu.eg \\ 3 Institute of Human Movement Science, Sport and Health, University of Graz, A-8010 Graz, Austria; \\ markus.tilp@uni-graz.at \\ 4 Faculty of Physical Education-Abo Qir, Alexandria University, Alexandria 21913, Egypt \\ * Correspondence: abdelrahman.akl@alexu.edu.eg
}

Citation: Salem, A.; Hassan, A.; Tilp, M.; Akl, A.-R. Antagonist Muscle Co-Activation during Kettlebell Single Arm Swing Exercise. Appl. Sci. 2021, 11, 4033. https://doi.org/ 10.3390/app11094033

Academic Editor: Zimi Sawacha

Received: 4 March 2021

Accepted: 23 April 2021

Published: 29 April 2021

Publisher's Note: MDPI stays neutral with regard to jurisdictional claims in published maps and institutional affiliations.

Copyright: (c) 2021 by the authors. Licensee MDPI, Basel, Switzerland. This article is an open access article distributed under the terms and conditions of the Creative Commons Attribution (CC BY) license (https:// creativecommons.org/licenses/by/ $4.0 /)$.

\begin{abstract}
The purpose of this study was to determine the muscle activation and co-activation of selected muscles during the kettlebell single arm swing exercise. To the best of our knowledge, this is the first study investigating the muscle co-activation of a kettlebell single arm swing exercise. Nine volunteers participated in the present study (age: $22.6 \pm 3.8$ years; body mass: $80.4 \pm 9.2 \mathrm{~kg}$; height: $175.6 \pm 7.5 \mathrm{~cm}$ ). The electrical muscle activity of eight right agonist/antagonist muscles (AD/PD, ESL/RA, ESI/EO, and GM/RF) were recorded using a surface EMG system (Myon m320RX; Myon, Switzerland) and processed using the integrated EMG to calculate a co-activation index (CoI) for the ascending and descending phases. A significant effect of the ascending and descending phases on the muscles' CoI was observed. Post hoc analyses showed that the co-activation was significantly higher in the descending phase compared to that in the ascending phase of AD/PD CoI $(34.25 \pm 18.03 \%$ and $24.75 \pm 13.03 \%, p<0.001), \mathrm{ESL} / \mathrm{RA} \mathrm{CoI}(34.97 \pm 17.86 \%$ and $24.19 \pm 10.32 \%, p<0.001), \mathrm{ESI} / \mathrm{EO} \mathrm{CoI}$ $(41.14 \pm 10.72 \%$ and $30.87 \pm 11.26 \%, p<0.001)$, and GM/RF CoI $(27.49 \pm 12.97 \%$ and $34.98 \pm 14.97 \%$, $p<0.001)$. In conclusion, the co-activation of the shoulder muscles varies within the kettlebell single arm swing. The highest level of co-activation was observed in the descending phase of $\mathrm{AD} / \mathrm{PD}$ and GM/RF CoI, and the lowest level of co-activation was observed during the descending phase, ESL/RA and ESI/EO CoI. In addition, the highest level of co-activation was observed in the ascending phase of ESL/RA and ESI/EO CoI, and the lowest level of co-activation was observed during the ascending phase, $\mathrm{AD} / \mathrm{PD}$ and GM/RF CoI. The co-activation index could be a useful method for the interpretation of the shoulder and core muscles' co-activity during a kettlebell single arm swing.
\end{abstract}

Keywords: kettlebell; antagonist muscle; electromyography; co-activation; movement analysis; injury prevention; wearable technologies

\section{Introduction}

In the last decade, there has been a significant increase in the popularity of kettlebell exercises [1-3]. Kettlebells have a unique design with an offset centre of gravity, which make them a remarkable all-in-one fitness method that combines strength training, cardiorespiratory conditioning, core stabilization, coordination, and dynamic mobility [1,2]. The kettlebell swing is one of the most frequently used kettlebell exercises and is one of the classical lifts that introduces the technical foundation of most other kettlebell exercises and forms the basis of many kettlebell exercise programs. The kettlebell swing can be performed with one or two arms $[1,3]$.

The movement requires ballistic flexion in the descending phase, followed by the ballistic extension of the hip joint in the ascending phase. In the ascending phase, the hip 
joint extends via the activation of the gluteus maximus and the hamstrings synergistically. Meanwhile, the shoulder joint flexes to $90^{\circ}$, supported by the activation of the anterior deltoid [4-6]. In the descending phase, the hip joint flexes via the activation of the gluteus maximus and the hamstrings eccentrically. Meanwhile, the shoulder joint extends, supported by the eccentric activation of anterior deltoid [1]. The coordinated recruitment of core muscles has a key role in trunk stabilization, allowing for an effective transfer of forces from the hip to the arms holding the kettlebell $[1,2,7]$.

Lake and Lauder [6] suggested that the mechanical demand of a kettlebell swing exercise appears to be sufficient to elicit increases in the ability to apply force quickly. Therefore, the kettlebell swing exercise could prove a useful addition to strength and conditioning practitioners interested in developing their athletes' ability to rapidly apply large forces to sports-specific resistances.

To our knowledge, there are only few studies that have investigated muscle activation via surface electromyography in the kettlebell swing exercise. Studies focused on the muscles most involved (such as the rectus abdominis, internal oblique, external oblique, lower and upper erector spinae, gluteus maximus, gluteus medius, biceps femoris, and anterior deltoid). Van Gelder, Hoogenboom, Alonzo, Briggs and Hatzel [5] showed that both a two-handed and single-handed kettlebell swing provide sufficient muscular recruitment for the strengthening of the gluteus maximus, gluteus medius, and biceps femoris. Single armed swings showed greater activation levels than two-armed swings, with greater activation on the contralateral side of the upper erector spinae and the ipsilateral side of the rectus abdominis [1]. Alternative kettlebell exercises (such as a kettlebell swing with kime (brief muscular pulsing at the top of the swing), kettlebell swing to kettlebell snatch, and kettlebell) are also effective exercises to strengthen the trunk muscles. McGill and Marshall [2]. Baek, Seol, Lee, Hong, Yu, and Kim [4] show that the activity of the biceps femoris, vastus medialis, erector spinae, and anterior deltoid depends on the shoulder joint flexion angle when conducting kettlebell swing.

Despite these interesting findings about the activity of agonist muscles during kettlebell exercises, the activation of antagonistic muscles that provide important information about the stability of the movement have so far been neglected. According to [8], the muscle co-activation is a simultaneous activation of two or more muscles around a joint, and it represents one of the central nervous system's action mechanisms that are responsible for joint stability. Therefore, the aim of this study was to determine the muscle activation and co-activation of selected muscles during the kettlebell single arm swing exercise. We hypothesized to identify different muscle-specific activations and different co-activation patterns in the ascending compared to the descending phase of the movement.

\section{Materials and Methods}

\subsection{Participants}

Nine participants volunteered in present study (age: $22.6 \pm 3.8$ years; body mass: $80.4 \pm 9.2 \mathrm{~kg}$; height: $175.6 \pm 7.5 \mathrm{~cm}$ ). The participant's consent was obtained and the study was conducted according to the Declaration of Helsinki and approved by the institutional ethics committee of studies and research.

\subsection{Experiment Protocol}

The participants were asked to perform a five-minute kettlebell-specific warm-up, with submaximal executions of the kettlebell swing exercise. Subsequently, participants performed three trials of one-arm kettlebell swings with five repetitions for each trial with a $16 \mathrm{~kg}$ kettlebell [2]. Between each trial, participants rested for one minute to avoid fatigue. The participants were instructed to lead the movement with the hips, flexing them during the descending phase, and extending them with maximal force and as quickly as possible during the ascending phase up to chest height (the "Russian swing") [9]. 


\subsection{Data Recordings}

The electrical muscle activity of eight right agonist/antagonist muscles (the anterior deltoid (AD), posterior deltoid (PD), erector spinae (longissimus) (ESL), rectus abdominis (RA), erector spinae (iliocostalis) (ESI), external oblique (EO), gluteus maximus (GM) and rectus femoris (RF)) were recorded using a surface EMG system (Myon m320RX; Myon, Switzerland). The skin over the muscles was shaved and cleaned with alcohol and bipolar, circular $10 \mathrm{~mm}$ diameter silver chloride surface electrodes (SKINTACT FSRG1/10, Leonhard Lang GmbH, Archenweg 56, 6020 Innsbruck, Austria) were secured on the selected muscles. Electrodes were attached over each muscle following the SENIAM guidelines maintaining a $2 \mathrm{~cm}$ centre-to-centre inter-electrode spacing [10]. The EMG signals were stored at a sampling frequency of $1000 \mathrm{~Hz}$ and digitized using a 16-bit analog to digital (A/D) converter. EMG data was processed using Visual 3D software (C-Motion, Germantown, MD, USA). Raw EMG data were band-pass $(20-450 \mathrm{~Hz})$ after applying a Butterworth filter. The signals were pre-processed using full-wave rectified and a linear envelope obtained using the Root Mean Square (RMS) approach. Data were normalized to an isometric maximum voluntary contraction (MVC), which was recorded after each subject finished the experimental tasks. To obtain the MVC values, subjects performed specific exercises for each muscle with three repetitions for $5 \mathrm{~s}$, with $60 \mathrm{~s}$ rest in between. Peak muscle activity over the three repetitions was taken as the MVC value.

\subsection{Co-Activation Index}

To analyse co-activation, the following pairs of muscles were investigated: agonist/antagonist muscles, AD/PD, ESL/RA, ESI/EO, and GM/RF. These pairs of muscles were selected because of their functions in stabilizing the shoulder joint, hip joint, and core. Muscle co-activation was estimated by the calculation of a co-activation index (CoI) using Equation (1) adapted from Kellis et al. [11]:

$$
\mathrm{CoI}=\frac{\mathrm{iEMG}_{\mathrm{anta}}}{\left(\mathrm{iEMG}_{\mathrm{anta}}+\mathrm{iEMG}_{\mathrm{ago}}\right)} \times 100
$$

where iEMGanta and iEMGago respectively refer to the normalized iEMG of the antagonist and agonist muscles in different movement phases. The ascending phase was defined as the lowest to the highest position of the kettlebell during the upward movement and the descending phase was defined as the highest to the lowest position of the kettlebell during the downward movement. These phases were determined with a video camera $(100 \mathrm{~Hz}$ frame rate, Basler scA640-120gc), which was synchronized with the EMG system. The electromyographical data of each movement repetition were then time-normalized for each duration of repetition.

The resulting CoI for the ascending and descending phases were calculated for each trial by averaging the five repetitions and the three trials for each participant.

\subsection{Statistical Analysis}

Descriptive statistics were reported as means and standard deviations (mean $\pm \mathrm{SD}$ ). The normality of the data was analysed using a Shapiro-Wilk test. A paired T-test was used to detect significant differences and compare the mean of each variable during the two phases (ascending and descending) of a single arm kettlebell swing. The statistical analysis was performed using IBM SPSS software Statistics v21.

\section{Results}

\subsection{Muscular Activity}

The average values and standard deviations for the normalized RMS for the AD and $\mathrm{PD}$ are presented in Figure 1 for the ascending and descending phases. Higher activities of the $\mathrm{AD}$ were observed during the ascending phase, with values of $22.44 \pm 10.15 \%$, compared to $15.75 \pm 6.50 \%$ during the descending phase. There was a significant difference 
between the $\mathrm{AD}$ activity during the ascending phase compared to the descending phase $(p<0.01$, Figure $1 b)$. For PD, slightly higher activities were observed during the descending phase (with values of $8.67 \pm 6.68 \%$ ), as compared to $7.54 \pm 6.00 \%$ during the ascending phase. The difference between the activity during the ascending and descending phases was not significant ( $p=0.155$, Figure $1 \mathrm{c})$.
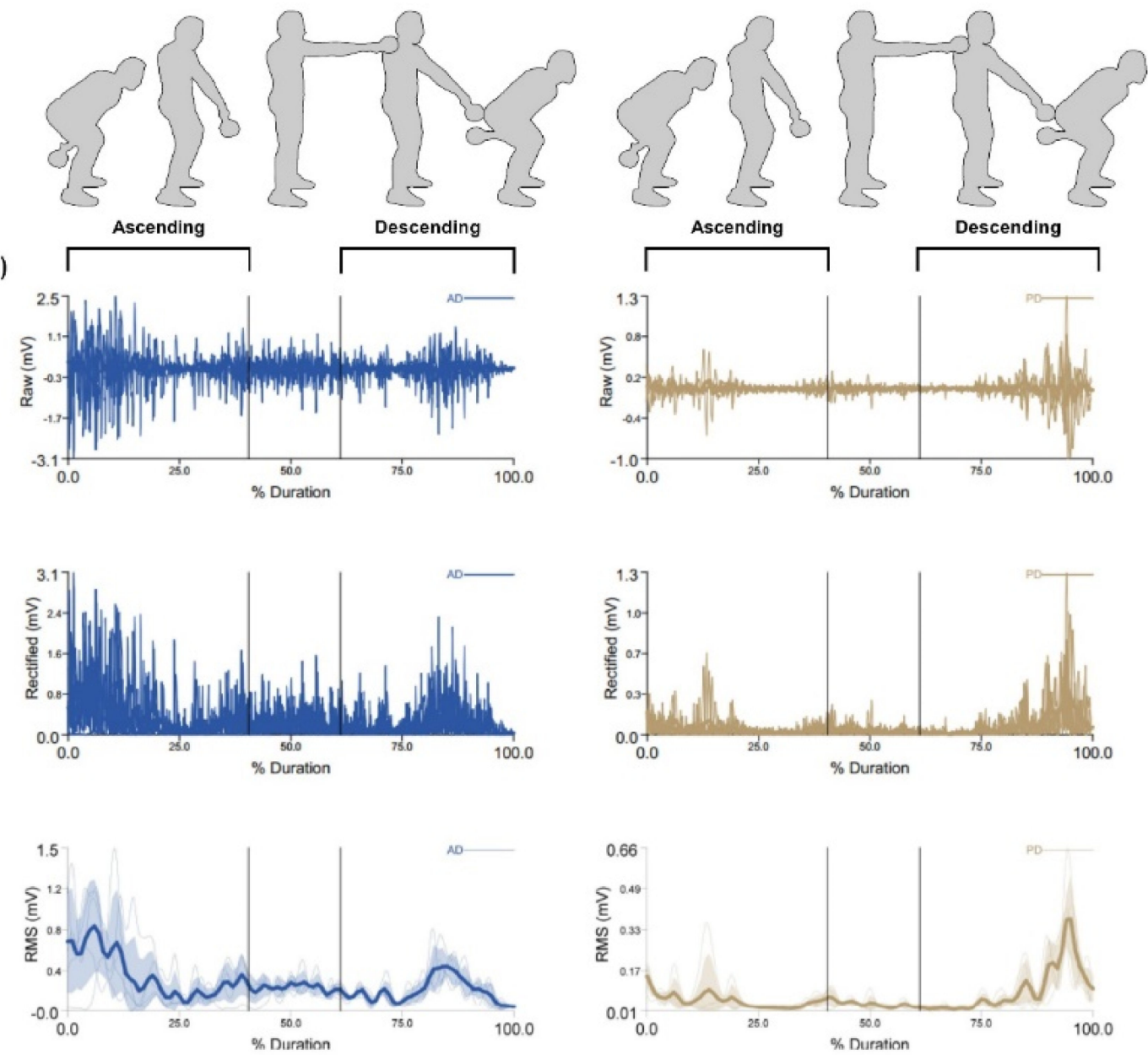

(b)

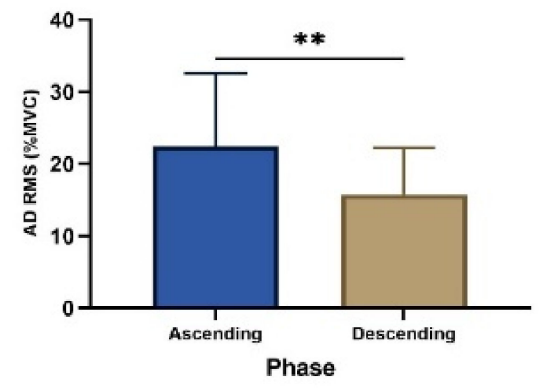

(c)

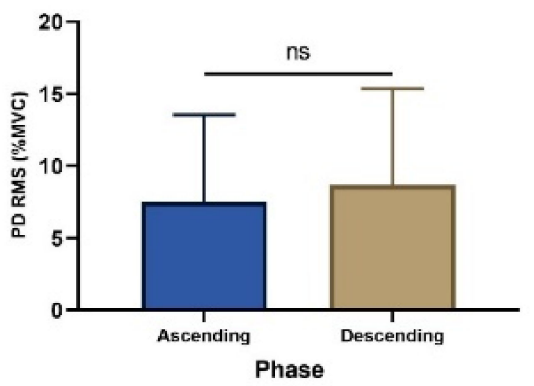

Anterior deltoid

Posterior deltoid

Figure 1. EMG data of the Anterior Deltoid (AD) and Posterior Deltoid (PD) during a kettlebell single arm swing (ascending and descending phases), (a) Exemplary EMG raw data, EMG rectified data, and EMG RMS from one representative trial. Average values and standard deviations for the normalised EMG (\%MVC) of the AD (b) and PD (c) during ascending and descending phases from all participants. Asterisk signs above the horizontal line represent significant differences between phases: $\left({ }^{* *}\right)$ indicates $p<0.01$ and (ns) indicates non-significant.

Average values and standard deviations for the normalized RMS for the ESL and RA are presented in Figure 2 for the ascending and descending phases. Higher activities of the ESL were observed during the ascending phase (with values of $19.27 \pm 9.62 \%$ ), 
as compared to $16.30 \pm 7.49 \%$ for the descending phase. There was a non-significant difference between the ESL activity during the ascending phase compared to that present during the descending phase ( $p=0.06$, Figure $2 b$ ). For RA, significantly higher activities $(p<0.001$, Figure 2c) were also observed during the ascending phase compared to those observed during the descending phase, with values of $10.51 \pm 7.23 \%$ and $5.22 \pm 3.28 \%$, respectively.

(a)
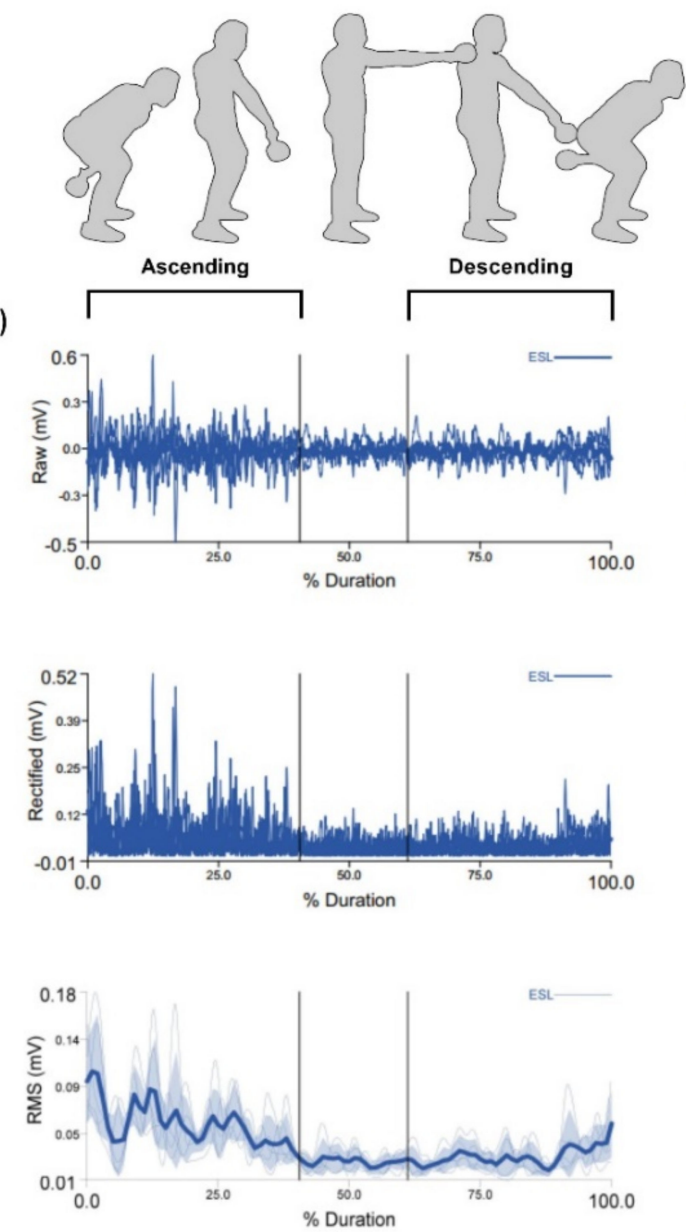

(b)

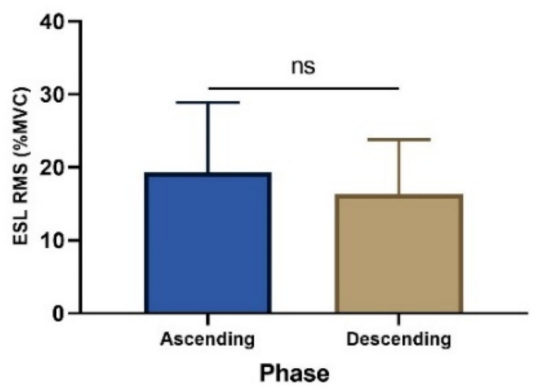

Erector spinae (Longissimus)
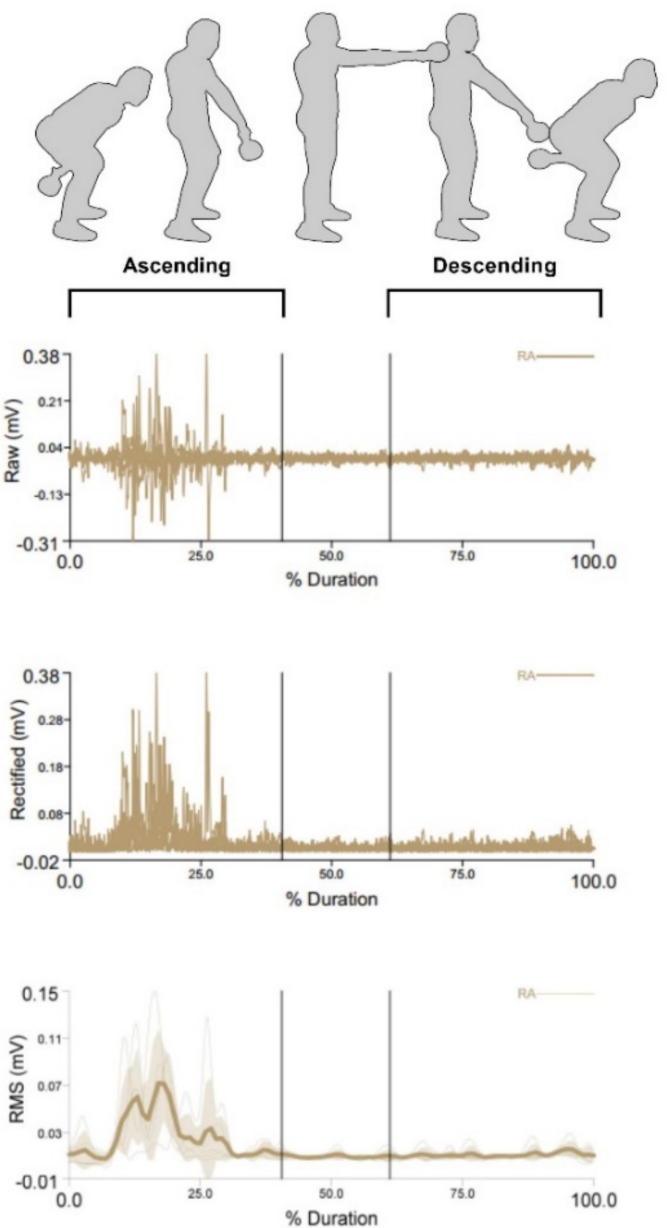

(c)

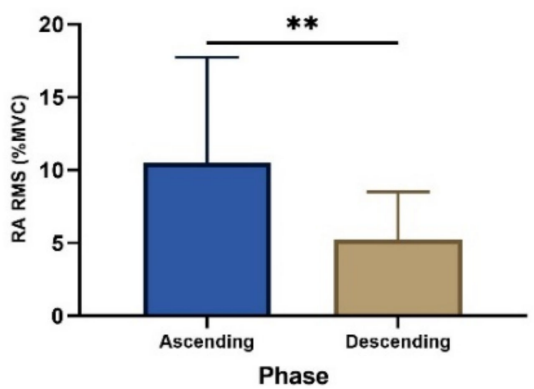

Rectus abdominis

Figure 2. EMG data of the erector spinae (longissimus) (ESL) and rectus abdominis (RA) during a kettlebell single arm swing (ascending and descending phases). (a) Exemplary EMG raw data, EMG rectified data, and EMG RMS from one representative trial. The average values and standard deviations for the normalised EMG (\%MVC) of the ESL (b) and RA (c) during ascending and descending phase from all participants. Asterisk signs above the horizontal line represent significant differences between the phases: $\left.{ }^{* *}\right)$ indicates $p<0.01$ and (ns) indicates non-significant.

Average values and standard deviations for the normalized RMS for the ESI and EO are presented in Figure 3 for the ascending and descending phases. Higher activities of the 
ESI were observed during the ascending phase than in the descending phase, with values of $21.75 \pm 10.97 \%$ and $19.94 \pm 8.21 \%$, respectively. This difference was not significant $(p=0.342$, Figure $3 b)$. For EO, significantly higher activities $(p<0.001$, Figure $3 c)$ were observed during the ascending phase compared to the descending phase, with values of $14.33 \pm 5.91 \%$ and $8.34 \pm 3.27 \%$, respectively.

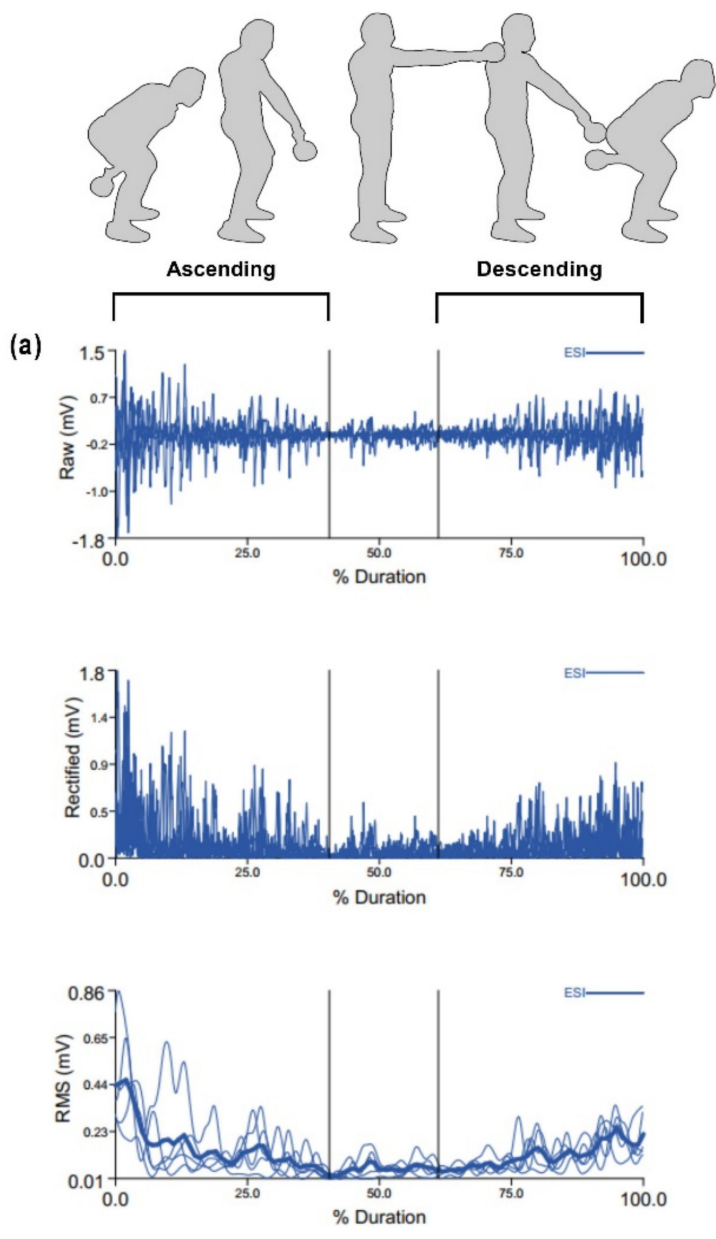

(b)

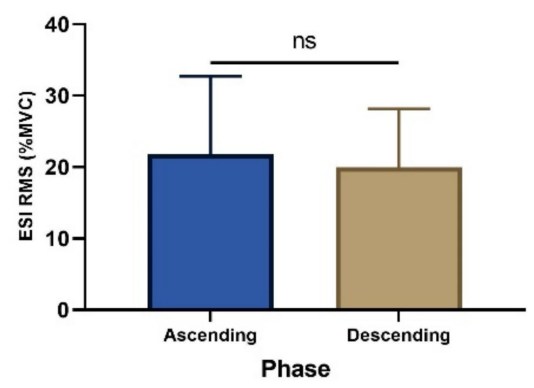

Erector spinae (lliocostalis)
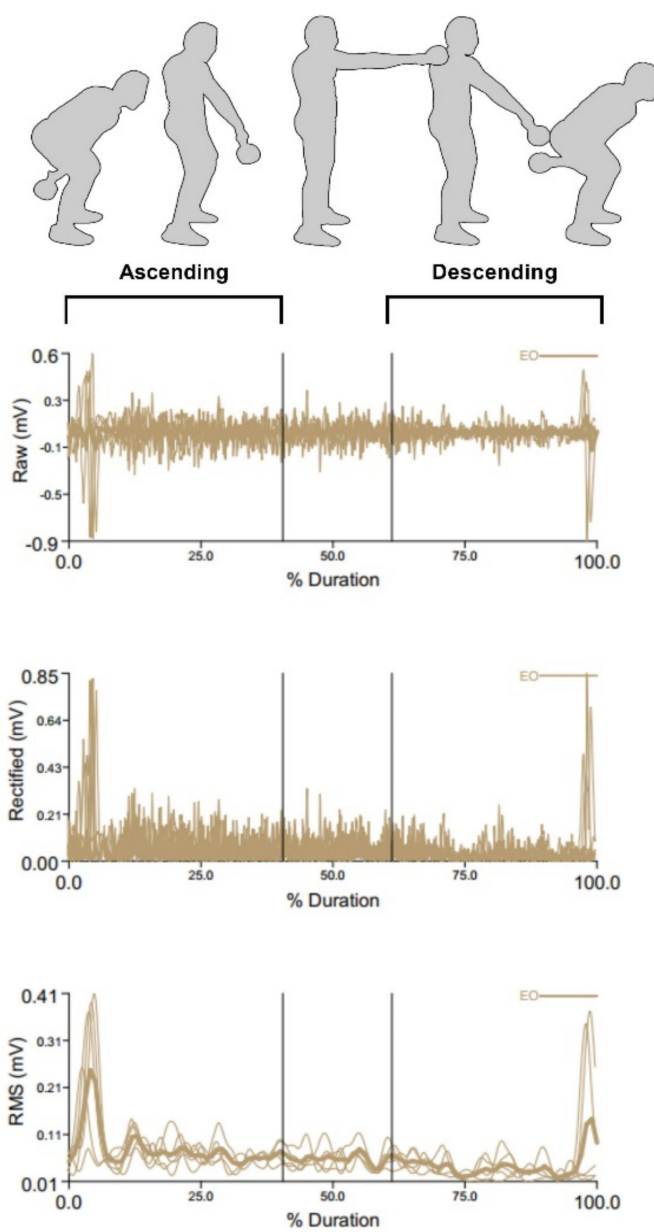

(c)

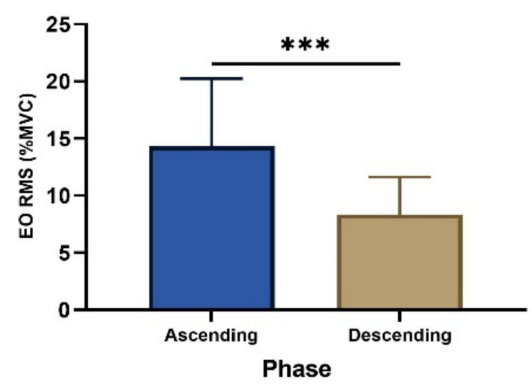

External Oblique

Figure 3. EMG data of the erector spinae (iliocostalis) (ESI) and external oblique (EO) during a kettlebell single arm swing (ascending and descending phases). (a) Exemplary EMG raw data, EMG rectified data, and EMG RMS from one representative trial. The average values and standard deviations for the normalised EMG (\%MVC) of the ESI (b) and EO (c) during the ascending and descending phase from all participants. Asterisk signs above the horizontal line represent significant differences between phases: ${ }^{* * *}$ indicates $p<0.001$ and (ns) indicates non-significant.

The average values and standard deviations for the normalized RMS for the GM and $\mathrm{RF}$ are presented in Figure 4 for the ascending and descending phases. Significantly higher 
GM activities were observed during the ascending phase than were observed during the descending phase $(p<0.001$, Figure $4 b)$, with values of $54.52 \pm 18.01 \%$ and $21.26 \pm 8.47 \%$, respectively. For $\mathrm{RF}$, significantly higher activities were also observed during the ascending phase than were observed during the descending phase $(p<0.001$, Figure $4 \mathrm{c})$, with values of $20.81 \pm 10.91 \%$ and $11.83 \pm 7.96 \%$, respectively.
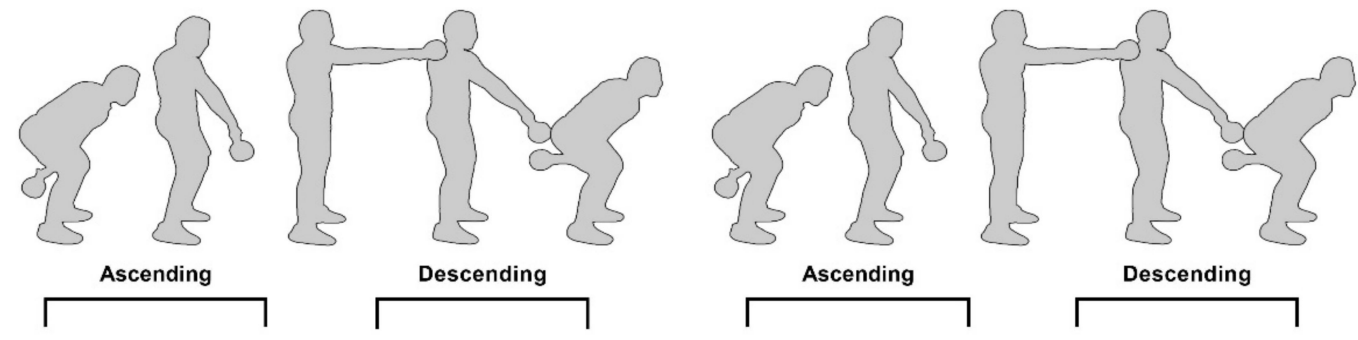

(a)
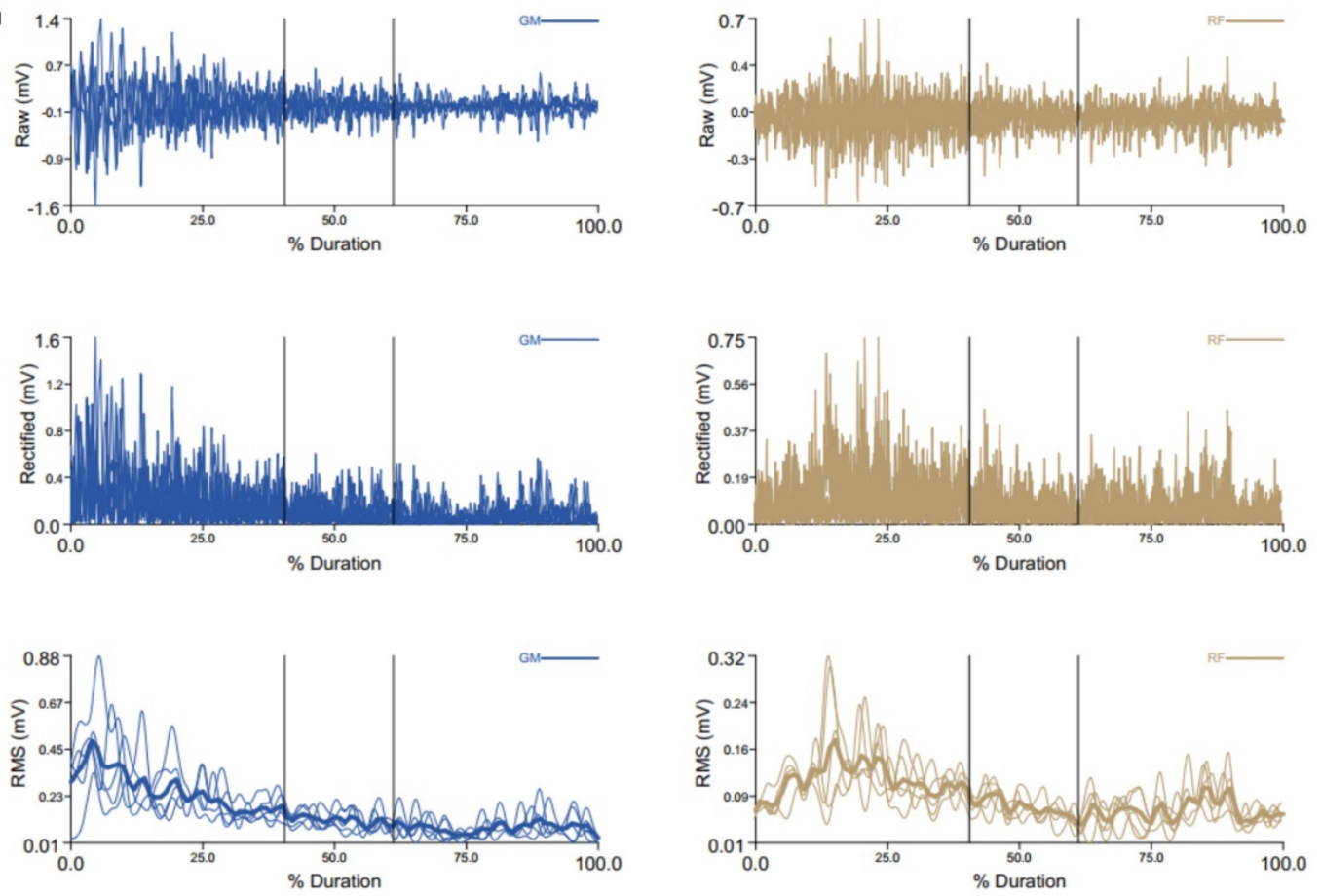

(b)

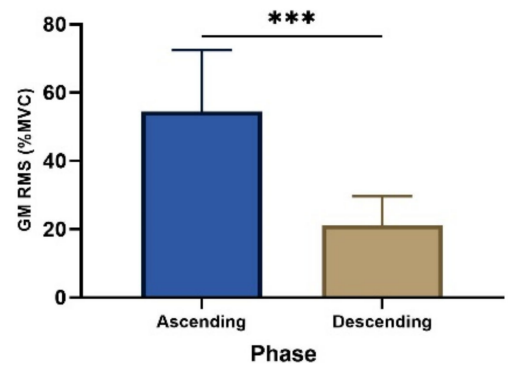

(c)

Gluteus Maximus

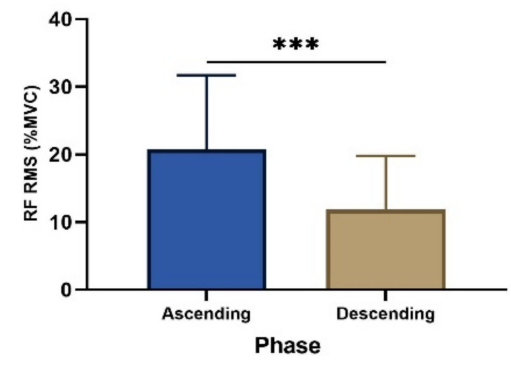

Rectus Femoris

Figure 4. EMG data of the gluteus maximus (GM) and rectus femoris (RF) during a kettlebell single arm swing (ascending and descending phases). (a) Exemplary EMG raw data, EMG rectified data, and EMG RMS from one representative trial. The average values and standard deviations for the normalised EMG (\%MVC) of the GM (b) and RF (c) during the ascending and descending phase from all participants. Asterisk signs above the horizontal line represent significant differences between phases: $\left({ }^{* * *}\right)$ indicates $p<0.001$. 


\subsection{Co-Activation Index}

Significant CoI differences between the ascending and descending phases in the investigated muscles pairs were observed (Figure 5). Post hoc analyses showed that the co-activation was significantly higher in the descending phase compared to that of the ascending phase for $\mathrm{AD} / \mathrm{PD}(34.25 \pm 18.03 \%$ vs. $24.75 \pm 13.03 \%, p<0.001)$ and $\mathrm{GM} / \mathrm{RF}$ $(34.98 \pm 14.97 \%$ vs. $27.49 \pm 12.97 \%, p<0.001)$. In contrast, the co-activation indices of ESL/RA $(34.97 \pm 17.86 \%$ vs. $24.19 \pm 10.32 \%, p<0.001)$ and ESI/EO $(41.14 \pm 10.72 \%$ vs. $30.87 \pm 11.26 \%, p<0.001$ ) were significantly higher in the ascending phase compared to the descending phase.

(a)

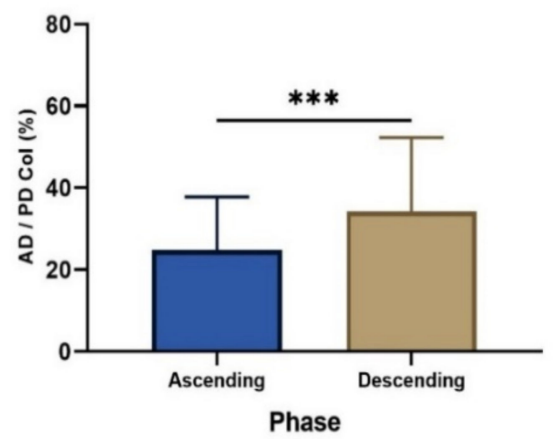

(c)

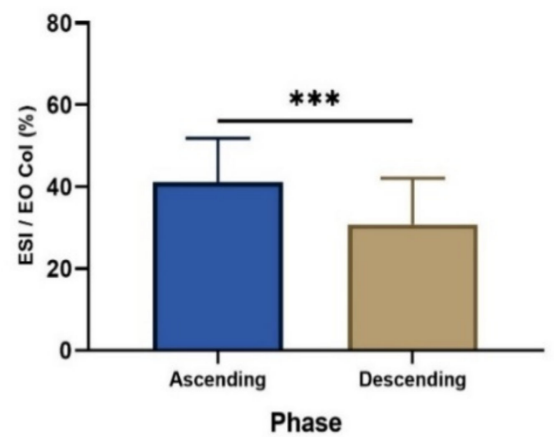

(b)

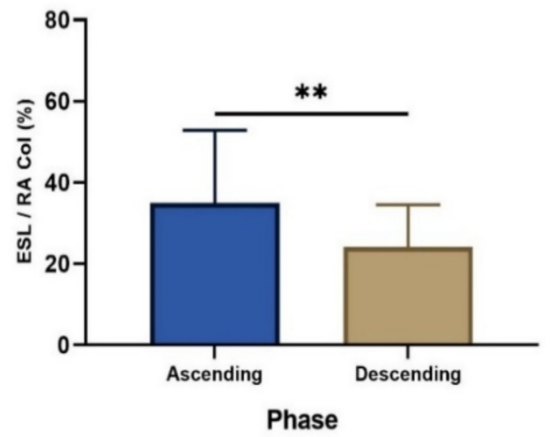

(d)

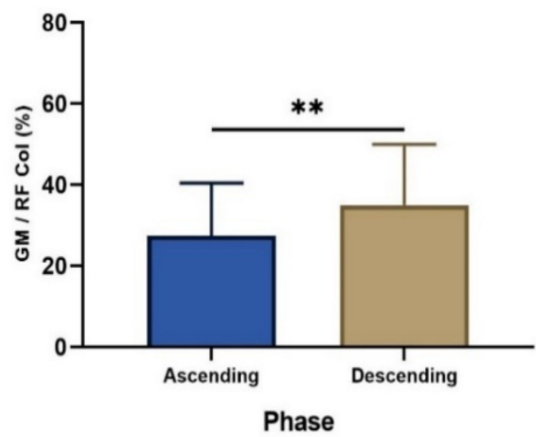

Figure 5. The average values and standard deviations for the CoI (\%) per phase. (a) AD/PD co-activation, (b) ESL/RA co-activation, (c) ESL/EO co-activation, and (d) GM/RF co-activation. Asterisk signs above the horizontal line represent significant differences between phases: $\left.{ }^{* * *}\right)$ indicates $p<0.001$ and $\left({ }^{* *}\right)$ indicates $p<0.01$.

\section{Discussion}

To our knowledge, this study is the first to quantify agonist-antagonist co-activation during single arm kettlebell swing exercise of the shoulder (AD/PD), core (ESL/RA, $\mathrm{ESI} / \mathrm{EO}$ ), and hip muscles (GM/RF). As hypothesized, the different agonist/antagonist muscle pairs showed significantly different co-activations in the ascending compared to the descending phase.

In the shoulder joint, the anterior deltoid showed greater activity $(\sim 20 \% \mathrm{MVC})$ than the posterior deltoid $(<10 \% \mathrm{MVC})$, especially during the ascending phase of the movement. This was expected, as the muscle is an agonist muscle during the flexion of the shoulder joint in the ascending phase. The activation of the anterior deltoid was specifically high during the first part of the upward movement to accelerate the kettlebell. When the kettlebell was close to its highest position, EMG activity decreased and increased again at the end of the downward movement to decelerate the movement of the kettlebell [12]. A simultaneous increase in the EMG activity of both muscles in the descending phase could 
be explained by the need for increased stability during the deceleration of the kettlebell, which is reached by increased co-activation [13].

The core muscles (erector spinae (longissimus) and rectus abdominis) both showed high activity in the ascending phase of the kettlebell swing. This was expected for the erector spinae (longissimus) $(<20 \% \mathrm{MVC})$, as it is responsible for producing the force to extend the trunk during the upward movement. The activity of the rectus abdominis ( $10 \%$ MVC) as an antagonist increases core stability, which supports the transfer of the forces from the hip extensors to the arms and the kettlebell. In addition, it may stabilize and protect the trunk during the explosive rotation movement of the one-armed swing exercise (Andersen, Fimland, Gunnarskog, Jungard, Slattland, Vraalsen, and Saeterbakken [1]). In particular, the generation of flexion moments results in the activation of the extensor core muscles $[14,15]$.

Furthermore, the activity of the erector spinae (longissiumus) ( 18\% MVC) in the descending phase is not surprising, since the activation of the extensor muscles is required to balance the flexion moment produced by the mass of the upper body. Generally, the activation of the erector spinae (longissimus) and the rectus abdominis is important in both phases because it mainly works to provide enough stability to prevent the spine from possible harmful twisting under load. The observed pattern of co-activation (which was greater in the ascending phase) might reflect the need to stabilize the trunk to transfer the forces from the hip extensors to the arms and kettlebell. This is in agreement with Brown and McGill [16] and Andersen, Fimland, Gunnarskog, Jungard, Slattland, Vraalsen, and Saeterbakken [1], who stated that the muscle co-activation is often substantial during trunk exertions.

The erector spinae (iliocostalis) is involved with almost the same activation in the ascending and descending phases ( 20\% MVC). The external oblique (which acts as antagonist eccentrically in the ascending phase) decelerates the spine extension and showed significant activation $(<15 \% \mathrm{MVC})$ in order to support the stability of the trunk and to avoid a rotation during the ascending phase. The well-coordinated contraction of the abdominal muscles provides protection for the spine. Because of the similar function, this pair of muscles acts similarly to the erector spinae (longissimus) and the rectus abdominis, with higher $\mathrm{CoI}$ during the explosive ascending phase. These results provide more information on the joint activation of muscles that act as both agonist and antagonist during trunk effort, with and without counting the additional resistance moment that must be overcome due to the joint activation of the muscles $[17,18]$.

In the lower limbs, the kettlebell swing emphasizes the activation of the gluteus maximus in the ascending phase ( $60 \% \mathrm{MVC})$, due to the nature of their action in bringing the leg in a straight line with the body and working to support the pelvis and make the body regain its erection after bending [19]. Furthermore, the gluteus maximus activity maintains the stability of the hip joint [19]. Van Gelder, Hoogenboom, Alonzo, Briggs, and Hatzel [5] indicate that higher gluteus maximus activity occurs during this phase while transitioning from hip flexion toward hip extension with the kettlebell accelerated in a pendular anterior/superior trajectory. In addition, the rectus femoris eccentrically decelerated the hip extension during the ascending (20\% MVC) phase and also showed considerable EMG activity ( $>10 \% \mathrm{MVC}$ ) during the descending phase, when it controls the hip flexion as an antagonist muscle to the gluteus maximus in the hip joint as well as decelerates the knee flexion together with the rest of the quadriceps group. The increase of rectus femoris activity leads to higher co-activation between GM/RF during the descending phase to control the hip flexion, with the kettlebell reversing curvilinear trajectory via the effect of gravity through forcible concentric contraction. The observed greater $\mathrm{CoI}$ during the descending phase is mainly due to the substantial reduction in activity of the gluteus maximus during the descending phase compared to the ascending phase from $54.52 \pm 18.01 \%$ to $21.26 \pm 8.47 \%$ MVC. Although the activity of the rectus femoris was also reduced from $20.81 \pm 10.91 \%$ and $11.83 \pm 7.96 \%$, the CoI increased significantly. 


\section{Conclusions}

In conclusion, the co-activation of the shoulder, core, and hip muscles varies during the different phases of the kettlebell single arm swing. Higher levels of co-activation were observed in the descending phase in the shoulder (AD/PD) and hip muscles (GM/RF). In contrast, the co-activation of the trunk muscles (ESL/RA and ESI/EO) was higher during the ascending phase of the movement. Hence, we conclude that great accelerations during the ascending phase are accompanied by increased co-activation of the trunk, possibly to improve force transmission from the legs to the arms and to protect the spine during this movement phase. During the descending phase, when the kettlebell is only accelerated by gravity, the subsequently lessened activity of the prime movers gluteus maximus and anterior deltoid (with a similar activity of their antagonists) led to an increase in co-activation in the hip and shoulder joint. The co-activation index was useful to interpret the shoulder, core, and hip muscles co-activity during a kettlebell single arm swing.

Author Contributions: Conceptualization, A.S. and A.-R.A.; methodology, A.-R.A. and A.S.; software, A.-R.A.; validation, A.-R.A.; A.H. and M.T.; formal analysis, A.-R.A.; A.H. and M.T.; investigation, A.-R.A.; resources, A.-R.A.; data curation, A.-R.A.; A.H. and M.T.; writing-original draft preparation, A.-R.A.; A.S.; A.H. and M.T.; writing-review and editing, A.-R.A.; A.H. and M.T.; visualization, A.-R.A.; A.H. and M.T.; supervision, A.-R.A.; project administration, A.-R.A. All authors have read and agreed to the published version of the manuscript.

Funding: This research received no external funding.

Institutional Review Board Statement: The study was conducted according to the guidelines of the Declaration of Helsinki and approved by the Ethics Committee of the Alexandria University.

Informed Consent Statement: Informed consent was obtained from all subjects involved in the study.

Data Availability Statement: The data presented in this study are available on request from the corresponding author.

Acknowledgments: The authors would like to thank the subjects for participating in this study.

Conflicts of Interest: The authors declare no conflict of interest.

\section{References}

1. Andersen, V.; Fimland, M.S.; Gunnarskog, A.; Jungard, G.A.; Slattland, R.A.; Vraalsen, O.F.; Saeterbakken, A.H. Core muscle activation in one-armed and two-armed kettlebell swing. J. Strength Cond. Res. 2016, 30, 1196-1204. [CrossRef] [PubMed]

2. McGill, S.M.; Marshall, L.W. Kettlebell swing, snatch, and bottoms-up carry: Back and hip muscle activation, motion, and low back loads. J. Strength Cond. Res. 2012, 26, 16-27. [CrossRef] [PubMed]

3. Jay, K.; Frisch, D.; Hansen, K.; Zebis, M.K.; Andersen, C.H.; Mortensen, O.S.; Andersen, L.L. Kettlebell training for musculoskeletal and cardiovascular health: A randomized controlled trial. Scand. J. Work Environ. Health 2011, 37, 196-203. [CrossRef] [PubMed]

4. Baek, H.-Y.; Seol, S.-J.; Lee, D.-Y.; Hong, J.-H.; Yu, J.-H.; Kim, J.-S. A comparison of muscle activity depending on shoulder joint flexion angle when conducting kettle bell swing. Med. Leg. Update 2020, 20, 1783-1787.

5. Van Gelder, L.H.; Hoogenboom, B.J.; Alonzo, B.; Briggs, D.; Hatzel, B. EMG analysis and sagittal plane kinematics of the two-handed and single-handed kettlebell swing: A descriptive study. Int. J. Sports Phys. Ther. 2015, 10, 811-826. [PubMed]

6. Lake, J.P.; Lauder, M.A. Mechanical demands of kettlebell swing exercise. J. Strength Cond. Res. 2012, 26, 3209-3216. [CrossRef] [PubMed]

7. Comerford, M.J.; Mottram, S.L. Functional stability re-training: Principles and strategies for managing mechanical dysfunction. Man. Ther. 2001, 6, 3-14. [CrossRef] [PubMed]

8. Yamazaki, Y.; Suzuki, M.; Ohkuwa, T.; Itoh, H. Coactivation in arm and shoulder muscles during voluntary fixation of a single joint. Brain Res. Bull. 2003, 59, 439-446. [CrossRef]

9. Meigh, N.J.; Keogh, J.W.L.; Schram, B.; Hing, W.A. Kettlebell training in clinical practice: A scoping review. BMC Sports Sci. Med. Rehabil. 2019, 11, 19. [CrossRef] [PubMed]

10. Hermens, H.J.; Freriks, B.; Disselhorst-Klug, C.; Rau, G. Development of recommendations for SEMG sensors and sensor placement procedures. J. Electromyogr. Kinesiol. 2000, 10, 361-374. [CrossRef]

11. Kellis, E.; Arabatzi, F.; Papadopoulos, C. Muscle co-activation around the knee in drop jumping using the co-contraction index. J. Electromyogr. Kinesiol. 2003, 13, 229-238. [CrossRef] 
12. Mitchell, J.; Johnson, W.M.; Riemann, B.; Krajewski, K.; Coates, C.W. Biomechanical Loading of the American Kettlebell Swing. In Proceedings of the ASME International Mechanical Engineering Congress and Exposition, Volume 3: Biomedical and Biotechnology Engineering, Houston, TX, USA, 13-19 November 2015. [CrossRef]

13. Franz, J.R.; Kram, R. How does age affect leg muscle activity/coactivity during uphill and downhill walking? Gait Posture 2013, 37, 378-384. [CrossRef] [PubMed]

14. Cholewicki, J.; McGill, S.M. Mechanical stability of the in vivo lumbar spine: Implications for injury and chronic low back pain. Clin. Biomech. 1996, 11, 1-15. [CrossRef]

15. Brown, S.H.; Potvin, J.R. Constraining spine stability levels in an optimization model leads to the prediction of trunk muscle cocontraction and improved spine compression force estimates. J. Biomech. 2005, 38, 745-754. [CrossRef] [PubMed]

16. Brown, S.H.; McGill, S.M. Co-activation alters the linear versus non-linear impression of the EMG-torque relationship of trunk muscles. J. Biomech. 2008, 41, 491-497. [CrossRef] [PubMed]

17. Lee, P.J.; Granata, K.P.; Moorhouse, K.M. Active trunk stiffness during voluntary isometric flexion and extension exertions. Hum. Factors 2007, 49, 100-109. [CrossRef] [PubMed]

18. Van Dieën, J.H.; Cholewicki, J.; Radebold, A. Trunk muscle recruitment patterns in patients with low back pain enhance the stability of the lumbar spine. Spine 2003, 28, 834-841. [CrossRef] [PubMed]

19. French, H.P.; Dunleavy, M.; Cusack, T. Activation levels of gluteus medius during therapeutic exercise as measured with electromyography: A structured review. Phys. Ther. Rev. 2010, 15, 92-105. [CrossRef] 